\title{
Endoscopic Third Ventriculostomy with Choroid Plexus Coagulation for Treatment of Hydrocephalus in X-linked Myotubular Myopathy: A Novel Approach
}

\author{
Adrienne Caiado, Abraham Schlauderaff ${ }^{2}$, Elias Rizk ${ }^{3}$ \\ 1. 2. Neurosurgery, Penn State Milton S. Hershey Medical Center 3. Department of Neurosurgery, Penn \\ State Hershey Medical Center
}

$\square$ Corresponding author: Abraham Schlauderaff , aschlauderaff@pennstatehealth.psu.edu Disclosures can be found in Additional Information at the end of the article

\section{Abstract}

X-linked myotubular myopathy (XLMTM) is a rare $\mathrm{x}$-linked disease. It manifests in male newborns with multiple comorbidities including severe hypotonia, absent deep-tendon reflexes, and respiratory weakness leading to ventilator dependence. The affected individuals usually die in early infancy but some individuals have survived into the second decade of life. Macrocephaly and hydrocephalus are common in XLMTM with some reporting it in up to $70 \%$ and $50 \%$ of cases, respectively. Hydrocephalus is usually communicating in nature and may require surgical intervention if it is progressive. Prior cases have also used ventriculoperitoneal shunts as the primary hydrocephalus treatment modality. Here we have described a case of treating XLMTM associated communicating hydrocephalus via endoscopic third ventriculostomy with choroid plexus coagulation. A 23-month-old male with XLMTM presented with macrocephaly and hydrocephalus. Previous cases of hydrocephalus in XLMTM were treated with the placement of a ventriculoperitoneal shunt. However, in this case, it was contraindicated due to extensive multisystem comorbities which restricted treatment options. Due to the lack of another feasible compartment for the placement of the cerebrospinal fluid (CSF) diversion, it was decided that an endoscopic third ventriculostomy with choroid plexus coagulation will be performed. During the patient's postoperative course, his head circumference stabilized at $50 \mathrm{~cm}$ and serial brain imaging indicated the decreasing size of the ventricular system. He also became more awake and interactive. His family withdrew care when he was 33-months-old, due to complications not related to the neurosurgery procedure. This case was the first time endoscopic third ventriculostomy with choroid plexus coagulation was successfully performed for the treatment of hydrocephalus in X-linked myotubular myopathy.

Received 08/23/2017 Review began 10/02/2017 Review ended 03/29/2018 Published 04/05/2018

C) Copyright 2018

Caiado et al. This is an open access article distributed under the terms of the Creative Commons Attribution License CC-BY 3.0., which permits unrestricted use, distribution, and reproduction in any medium, provided the original author and source are credited.
Categories: Pediatric Surgery, Neurosurgery

Keywords: x-linked myotubular myopathy, myotubular myopathy, endoscopic third ventriculostomy, choroid plexus coagulation, hydrocephalus, pediatric

\section{Introduction}

X-linked myotubular myopathy (XLMTM) is a severe, rare x-linked neuromuscular disorder of the centronuclear myopathies family. XLMTM occurs when there is a mutation in the gene which encodes the protein tyrosine phosphatase (myotubularin protein, MTM1), which is required for muscle cell differentiation [1]. It presents in male new-borns with multiple comorbidities including severe hypotonia, absent deep-tendon reflexes, impaired bulbar 


\section{Cureus}

function (leading to feeding difficulties), and respiratory weakness (leading to ventilator dependence). Affected individuals usually die in early infancy, but some survive into the second decade of life. Myopathic changes are seen on electromyography (EMG) and the diagnosis is confirmed by muscle biopsy demonstrating muscle fibers with one or more centrally-placed nuclei, with a surrounding clear area due to the absence of myofibrils [1]. Macrocephaly and hydrocephalus are common in XLMTM with some reporting it in up to $70 \%$ and $50 \%$ of cases, respectively [2-3]. Hydrocephalus is typically communicating in nature and may require surgical intervention if progressive. Prior cases have used ventriculoperitoneal shunts as the primary hydrocephalus treatment modality [2-3]. Here, we have described a case of treating XLMTM-associated communicating hydrocephalus via endoscopic third ventriculostomy, with choroid plexus coagulation (ETV/CPC).

\section{Case Presentation}

A 23-month-old male with XLMTM presented with macrocephaly and hydrocephalus. The patient's magnetic resonance imaging (MRI), obtained prior to surgical intervention (Figure 1), demonstrated progressive diffuse parenchymal volume loss compared to an MRI performed six days following birth.

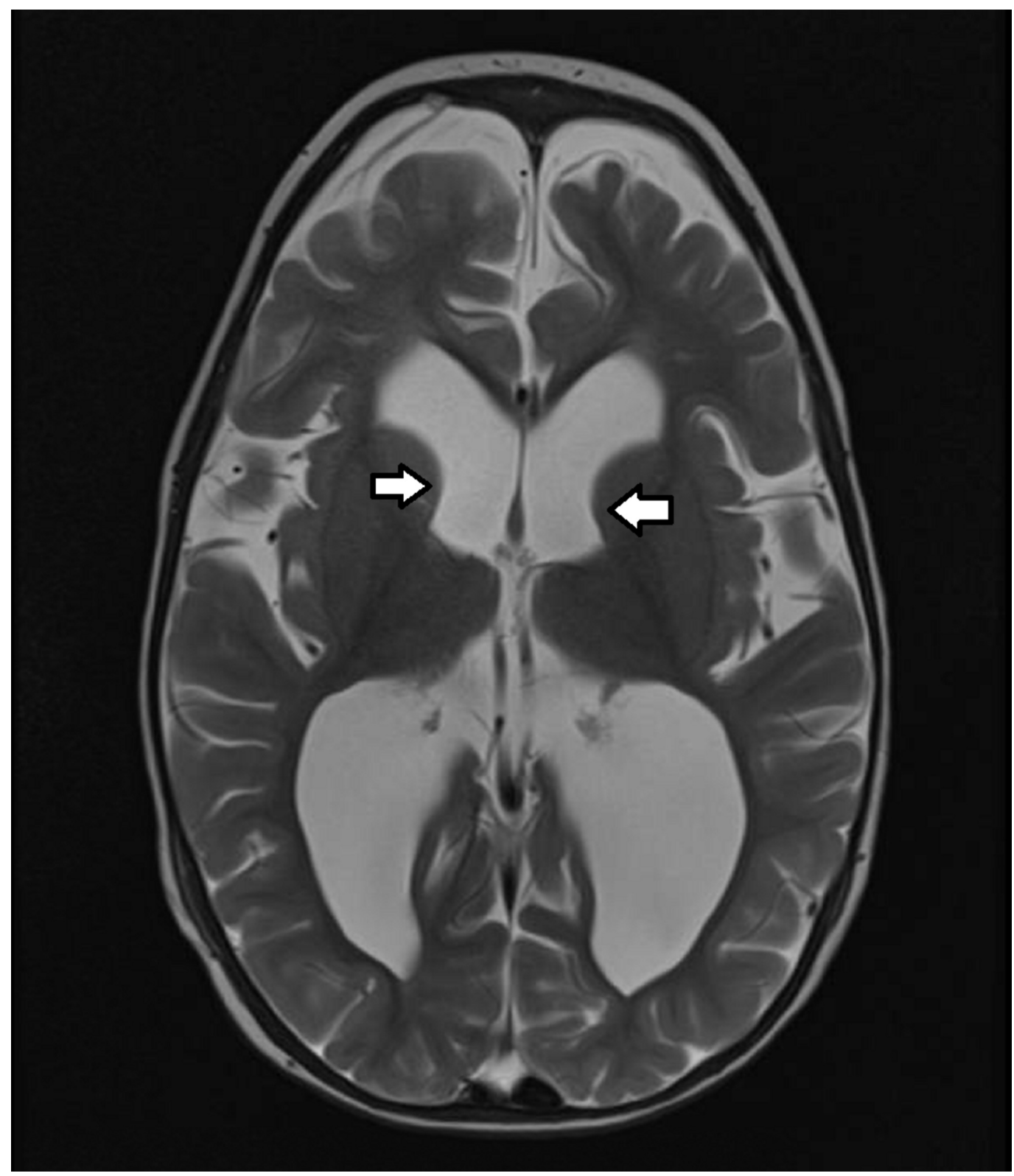


FIGURE 1: MRI prior to ETVICPC

Ventriculomegally is noted. Arrows point to bilaterally enlarged frontal horns.

MRI: magnetic resonance imaging; ETV/CPC: endoscopic third ventriculostomy, with choroid plexus coagulation.

In particular, there was extensive central white matter volume loss, with prominent enlargement of the ventricular system. The degree of ventriculomegaly was more than expected for volume loss, indicating a component of communicating hydrocephalus. The child's head circumference was initially at the 50th percentile for age at birth. Over time, the child's head circumference growth accelerated disproportionate for his age, progressing to $>99$ th percentile.

Previous cases of hydrocephalus in XLMTM have been treated with the placement of a ventriculoperitoneal shunt [2-4]. However, the placement of such a ventriculoperitoneal shunt was contraindicated in this case due to extensive multisystem comorbidities including chronic respiratory and hepatic failure. Without an alternative compartment for the placement of a cerebrospinal fluid (CSF) diversion, we performed an endoscopic third ventriculostomy, septostomy, and bilateral choroid plexus coagulation at the age of 26 months.

During the patient's postoperative course, his head circumference stabilized at $50 \mathrm{~cm}$ and serial brain imaging indicated the decreasing size of the ventricular system (Figures 2-3). 


\section{Cureus}

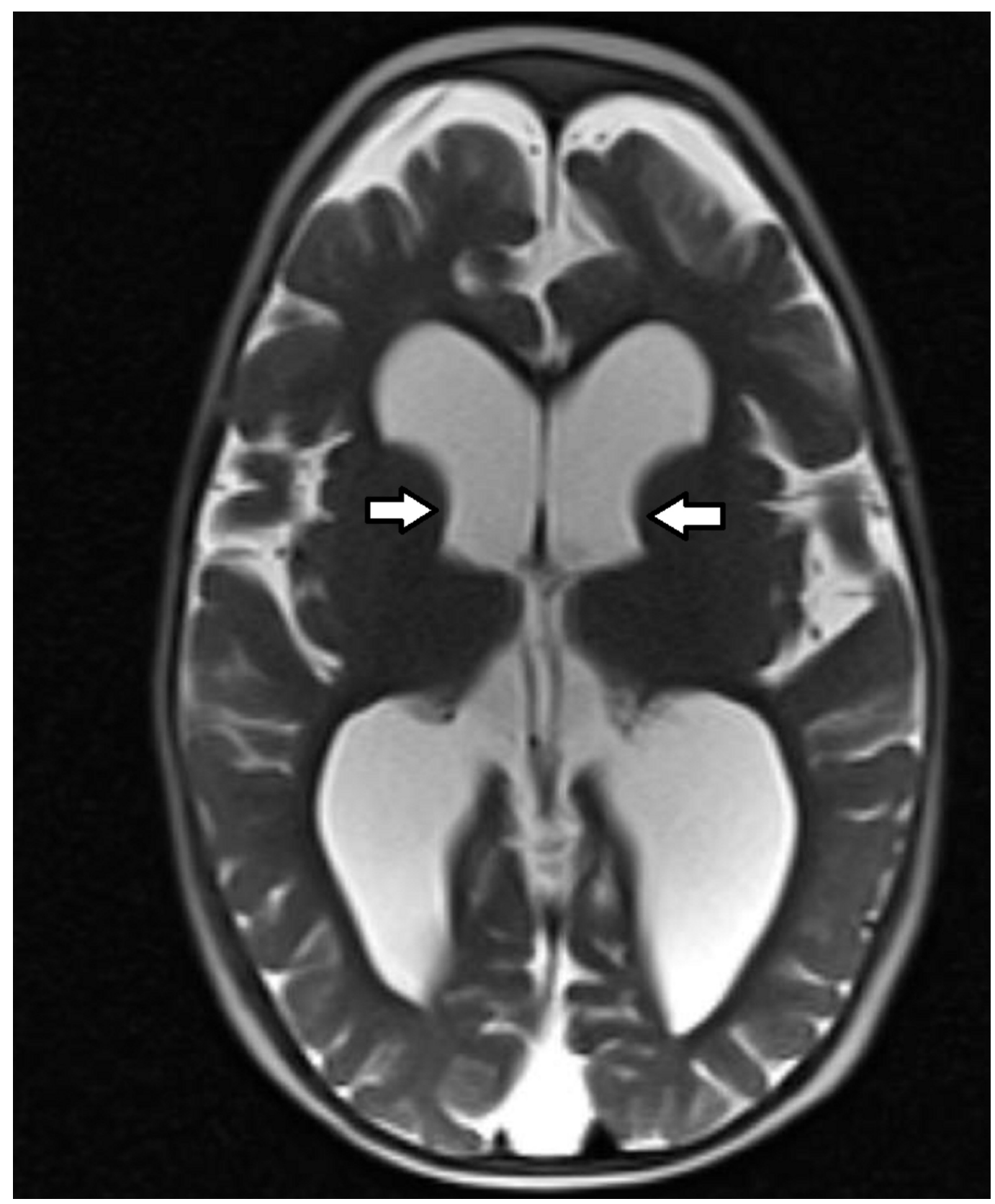

FIGURE 2: Post-operate MRI, at last follow-up

MRI at last postoperative follow-up prior to patient passing away from other system causes which shows stable ventricular size (arrows again pointing to enlarged frontal horns).

MRI: Magnetic resonance imaging 


\section{Cureus}

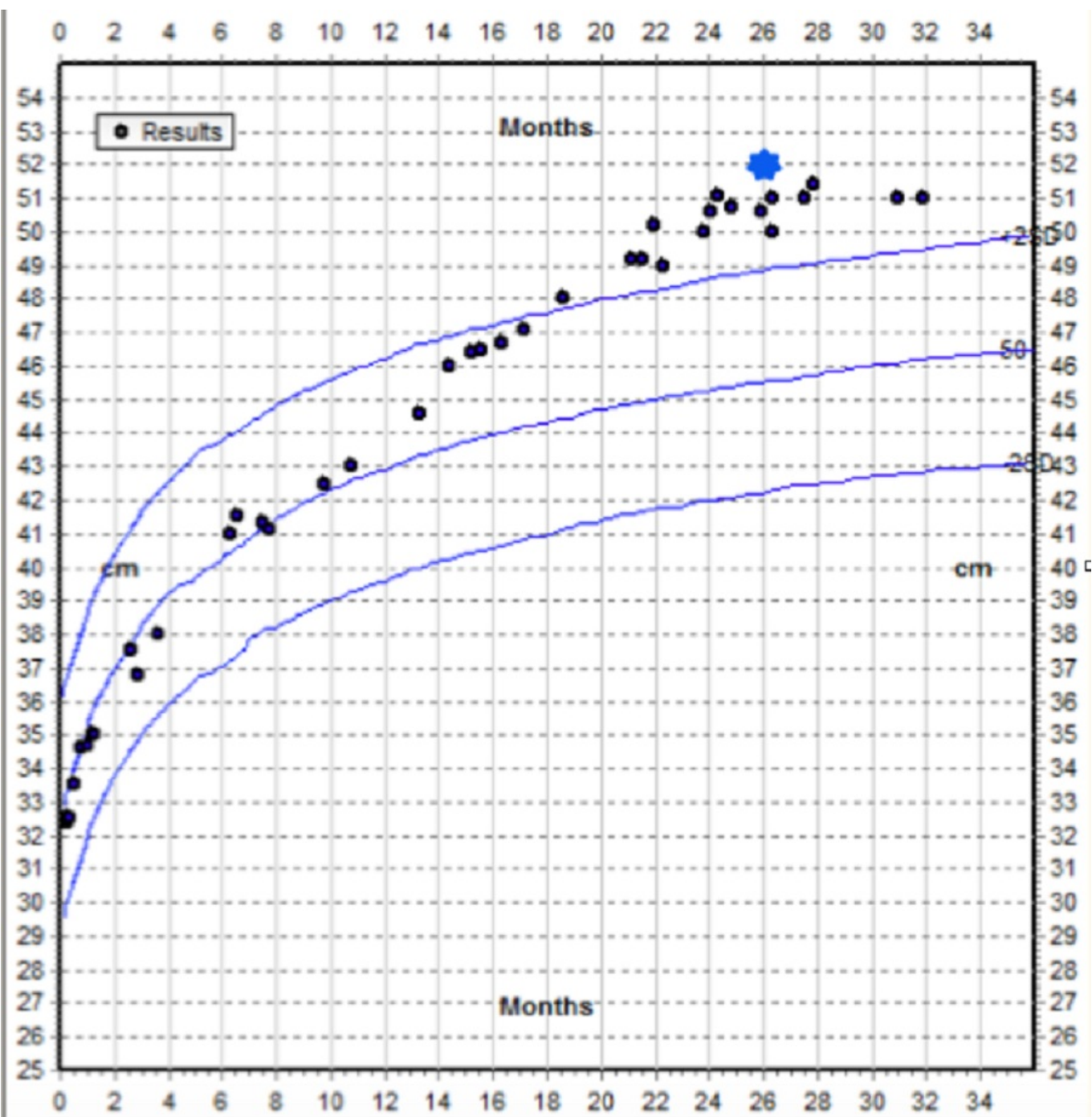

\section{FIGURE 3: Head circumference graph}

Depicted here is head circumference (in centimeters on the Y-axis) over time (in months on the $\mathrm{X}$-axis). Prior to the ETV and CPC being performed at 26 weeks (blue star) there was progressive ventriculomegaly crossing percentile lines. After the surgery there was stabilization of head circumference until last follow up.

He also became more alert and interactive. Ultimately, the patient's family withdrew care when he was 33-months-old, due to complications from end-stage liver failure.

\section{Discussion}

When rapidly progressive hydrocephalus is identified, intervention must be considered as it can lead to craniocerebral disproportion, as in this patient. Such disproportion can lead to-if not sudden death-reduced quality of life due to several factors, including irritability, failure of head control, and problems with head positioning [5]. The current surgical intervention recommended for rapidly progressive hydrocephalus in children is either a CSF shunt such as a ventriculoperitoneal shunt or endoscopic third ventriculostomy [2].

In the literature review, the most extensive studies of combined ETV/CPC have been done in infants less than 24-months-old, primarily in developing countries with hydrocephalus. The 
majority of studies appear to be in sub-Saharan Africa and have demonstrated that combined ETV/CPC is significantly superior to ETV alone in treating aqueductal stenosis, myelomeningocele, or Dandy-Walker complex [5-6]. One study performed on infants younger than one year of age with hydrocephalus due to congenital aqueductal stenosis demonstrated 81.9\% survival following ETV/CPC as compared to 48.6\% survival with ETV alone. This same study reported that all known treatment failures occurred within 6.2 months of surgery [5]. If this same time frame is applied to this case, our patient survived without treatment failure to roughly seven months, thus past the point of treatment failure expected. Another study performed on a larger patient population reported a $66 \%$ success rate for ETV/CPC compared to $47 \%$ with ETV alone in patients less than one year of age [6]. It has been demonstrated that supplementing ETV with CPC decreases the likelihood of eventual shunt dependence by about $20 \%$ [7]. In a retrospective review of seven Hydrocephalus Clinical Research Network centers in North America, ETV/CPC in infants under two years of age with hydrocephalus was found to be reasonably safe [8].

\section{Conclusions}

This was the first successful case using endoscopic third ventriculostomy with choroid plexus coagulation for the treatment of hydrocephalus in X-linked myotubular myopathy. The limitation of this report is that this example of ETV/CPC was performed on one patient with a rare congenital condition, of which little is known. This patient's case was also unique given that the etiology of hydrocephalus could not be explained by a more understood condition, such as aqueductal stenosis, myelomeningocele, or Dandy-Walker malformation. In addition, followup of the success of this procedure was limited by the unfortunate death of the patient, due to non-neurological sequelae of XLMTM. Future research needs to focus on understanding the relationship between XLMTM and hydrocephalus, as well as long-term follow-up of patients undergoing ETV/CPC.

\section{Additional Information}

\section{Disclosures}

Human subjects: Consent was obtained by all participants in this study. Conflicts of interest: In compliance with the ICMJE uniform disclosure form, all authors declare the following: Payment/services info: All authors have declared that no financial support was received from any organization for the submitted work. Financial relationships: All authors have declared that they have no financial relationships at present or within the previous three years with any organizations that might have an interest in the submitted work. Other relationships: All authors have declared that there are no other relationships or activities that could appear to have influenced the submitted work.

\section{References}

1. Lawlor MW, Beggs AH, Buj-Bello A, et al.: Skeletal muscle pathology in x-linked myotubular myopathy: review with cross-species comparisons. J Neuropathol Exp Neurol. 2016, 75:102110. 10.1093/jnen/nlv020

2. Herman GE, Finegold M, Zhao,W, et al.: Medical complications in long-term survivors with Xlinked myotubular myopathy. J Pediatr. 1999, 134:206-214. 10.1016/S0022-3476(99)70417-8

3. Joseph M, Pai GS, Holden KR, Herman G: X-linked myotubular myopathy: clinical observations in ten additional cases. Am J Med Genet A. 1995, 59:168-173.

10.1002/ajmg.1320590211

4. Congenital Myopathies. (2017). Accessed: August 23, 2017: https://www.uptodate.com/contents/congenital-myopathies.

5. Kulkami AV, Riva-Cambrin J, Browd SR, et al.: Endoscopic third ventriculostomy and choroid plexus cauterization in infants with hydrocephalus: a retrospective Hydrocephalus Clinical Research Network study. J Neurosurg Pediatr. 2014, 14:224-229. 10.3171/2014.6.PEDS13492 


\section{Cureus}

6. Warf BC: Comparison of endoscopic third ventriculostomy alone and combined with choroid plexus cauterization in infants younger than 1 year of age: a prospective study in 550 African children. J Neurosurg Pediatr. 2012, 116:475-481.

7. Warf BC, Tracy S, Mugamba J: Long-term outcome for endoscopic third ventriculostomy alone or in combination with choroid plexus cauterization for congenital aqueductal stenosis in African infants. J Neurosurg Pediatr. 2012, 10:108-111. 10.3171/2012.4.PEDS1253

8. Dewan MC, Naftel RP: The global rise of endoscopic third ventriculostomy with choroid plexus cauterization in pediatric hydrocephalus. Pediatr Neurosurg. 2016, 52:401-408. $10.1159 / 000452809$ 